\title{
ApoE receptor 2: a double-edged sword
}

DOI:

$10.1038 / \mathrm{nrn} 2081$
Carrying the apolipoprotein E4 (ApoE4) allele increases the risk of developing Alzheimer's disease, but the role of ApoE receptors in the disease process is unclear. Focusing on the ApoE receptor 2 (ApoE-R2), Beffert et al. showed that it regulates neuronal survival both during ageing and after injury, and that this requires the presence of the alternatively spliced exon 19.

Using retrograde fluorescent-dye labelling of corticospinal neurons to compare neuronal survival in mice lacking ApoE-R2 and wild-type mice, the authors showed that all mice had equal numbers of corticospinal neurons at 1 month of age, indicating similar levels of neurogenesis. Three months later, ApoE- $2^{-/-}$mice had lost half the labelled neurons, whereas control mice had lost none. By contrast, when corticospinal neurons were damaged by stereotaxic deafferentiation at the level of the internal capsule, no neurons died in adult ApoE-R2 ${ }^{-/-}$mice, whereas control animals lost $40 \%$ of the damaged neurons. Therefore, ApoE-R2 protects against neuronal loss that is associated with ageing, but promotes neuronal death after injury.

The intracellular domain of ApoE-R2 contains the alternatively spliced exon 19, which binds to, among others, Jun N-terminal kinase-interacting proteins (JIPs).
Using transgenic mice the authors next aimed to establish the involvement of this exon in the ability of ApoE-R2 to regulate neuronal survival. Eight-month-old mice lacking exon 19 had 36\% fewer corticospinal neurons compared with controls, as well as a significant reduction in the thickness of their primary motor cortex. By contrast, after neuronal injury these mice displayed no neuronal loss, whereas mice expressing exon 19 had lost $40 \%$ of neurons.

Together, these findings indicate that exon 19, the ApoE-R2 domain that recruits JIPs, is crucial for the regulation of neuronal survival during ageing and after injury.

JIPs bound to exon 19 of ApoE-R2 interact with Jun $\mathrm{N}$-terminal kinases (JNKs). The researchers found that JNK3, which is found preferentially in neurons and is activated after neuronal lesions, is required for the death-promoting role of ApoE-R2 after neuronal injury: $\mathrm{JNK}^{-/-}$mice had normal baseline numbers of corticospinal neurons at 4 months of age, and did not exhibit neuronal death after injury.

It is possible that the exon 19-containing isoform of ApoE-R2 protects against ageing-associated neuronal death by keeping the assembly of JNK signalling complexes inactive at the neuronal membrane, but that after injury the complexes might be dissociated from the membrane and become death-promoting. The results also suggest that the effects of different ApoE alleles on neurodegeneration could be dependent on the isoform of their receptors.

Leonie Welberg

ORIGINAL RESEARCH PAPER Beffert, U. et al. ApoE receptor 2 controls neuronal survival in the adult brain. Curr. Biol. 16, 2446-2452 (2006)

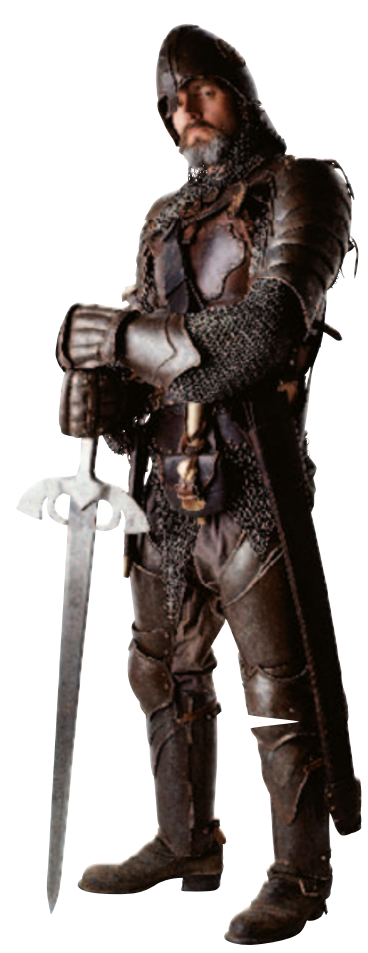

